\title{
A Stratified Linear Sound Speed Profile Simplification Method for Localization Correction
}

\author{
Wei Huang \\ scholarhw@gmail.com \\ School of Electronic Information \\ Wuhan University \\ Wuhan, Hubei, China \\ Collaborative Innovation Center of \\ Geospatial Technology \\ Wuhan, Hubei, China
}

\author{
Mingliu Liu* \\ Deshi Li \\ liumingliu@whu.edu.cn \\ dsli@whu.edu.cn \\ School of Electronic Information \\ Wuhan University \\ Wuhan, Hubei, China
}

\author{
Yi Cen \\ Shuaijun Wang \\ cenyi@whu.edu.cn \\ wangsj@whu.edu.cn \\ School of Electronic Information, \\ Wuhan University \\ Wuhan, Hubei, China
}

\begin{abstract}
Localization is important for underwater sensor networks as the validity of data and the maintenance of nodes both need location information. Because of the Snell effect caused by non-uniform distribution of sound speed, the signal propagation path is curved, which would lead to errors when traditional linear propagation localization models are used. Sound speed profiles (SSPs) can be used to correct the signal trajectory error according to the ray theory, so as to improve the localization accuracy. To simplify the expression of original SSPs and reduce the computational complexity of ray tracing while guaranteeing the accuracy, we propose a stratified linear SSP simplification method with a distance-minimization-based equal-interval control points searching (DM-EICPS) algorithm. Simulation results show that, compared with other curve approximation algorithms, the DM-EICPS algorithm not only generates simplified SSPs rapidly, but also achieves good SSP approximation accuracy. Moreover, the corrected localization accuracy with simplified SSPs is an order of magnitude higher than the linear propagation model, and the time overhead of ray tracing with simplified SSPs is obviously reduced compared to original SSPs.
\end{abstract}

\section{KEYWORDS}

distance-minimization, control points, equal-interval searching, localization correction, sound speed profile (SSP)

\section{ACM Reference Format:}

Wei Huang, Mingliu Liu, Deshi Li, Yi Cen, and Shuaijun Wang. 2019. A Stratified Linear Sound Speed Profile Simplification Method for Localization Correction. In WUWNET'19: International Conference on Underwater Networks \& Systems (WUWNET'19), October 23-25, 2019, Atlanta, GA, USA. ACM, New York, NY, USA, 6 pages. https://doi.org/10.1145/3366486.3366517

\footnotetext{
${ }^{*}$ Corresponding author.
}

The authors would like to thank the National Oceanic and Atmospheric Administration (https://www.nodc.noaa.gov) for providing the CTD database. The work is supported by the National Natural Science Foundation of China under Grant No. 61571334

Permission to make digital or hard copies of part or all of this work for personal or classroom use is granted without fee provided that copies are not made or distributed for profit or commercial advantage and that copies bear this notice and the full citation on the first page. Copyrights for third-party components of this work must be honored

For all other uses, contact the owner/author(s).

WUWNET'19, October 23-25, 2019, Atlanta, GA, USA

(c) 2019 Copyright held by the owner/author(s).

ACM ISBN 978-1-4503-7740-9/19/10.

https://doi.org/10.1145/3366486.3366517

\section{INTRODUCTION}

In recent years, the exploitation and utilization requirement of marine resources has grown rapidly, with the development of underwater acoustic communication technologies and embedded systems, underwater acoustic sensor networks (UWSNs) have become an effective means of monitoring the ocean environment. Localization is essential for UWSNs because the validity of data and the maintenance of nodes both need location information.

Some underwater acoustic localization algorithms based on received signal strength indication (RSSI) or time of arrival (TOA) information are proposed by researchers $[1,2,4,11,14]$, where the sound speed is usually assumed to be constant, and the acoustic signal is considered to be traveling along a straight line. However, the real sound speed is non-uniformly distributed as a function of temperature, salinity and static pressure, which can be expressed by the Medwin formula [6]. Urick [10] points out that the ocean sound speed distribution has obvious stratification characteristics with depth, and it is usually described by sound speed profiles (SSPs). Because of the Snell refraction effect caused by variable sound speed, the bending of sound waves will have a non-negligible impact on underwater acoustic positioning.

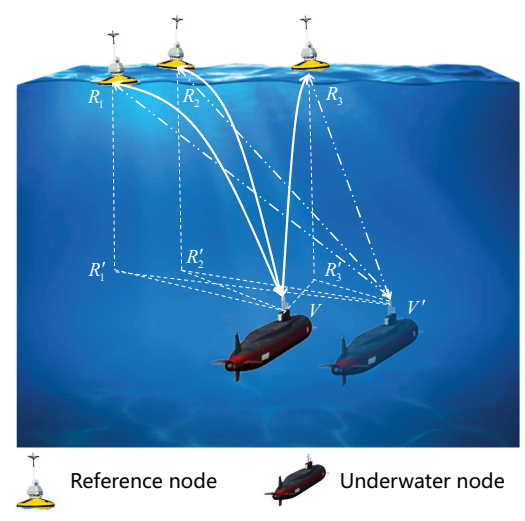

Figure 1: The influence of varying sound speed on underwater acoustic localization.

As the depth can be measured by an equipped pressure sensor, the successful localization of a node requires at least three reference locations. Fig. 1 gives an example to illustrate the effect of variable sound speed on positioning, where $R_{1}, R_{2}, R_{3}$ are three reference 
buoy nodes that can be directly located through the global positioning system (GPS), $V$ is the real position of a target node to be located, $R_{1}^{\prime}, R_{2}^{\prime}, R_{3}^{\prime}$ are the corresponding projection points of the reference nodes, the solid curves represent the real path of acoustic waves, $V^{\prime}$ is the located position of the target node obtained by a linear propagation model of acoustic waves. When the actual signal propagation time along the curve is utilized in the linear propagation localization model, there will be some ranging errors between the estimated node distance $V^{\prime} R_{n}, n=1,2,3$ and the real node distance $V R_{n}, n=1,2,3$, which will reduce the localization accuracy. If the real sound speed distribution is obtained, the measured distance can be corrected through the ray tracing theory, and finally the localization accuracy can be improved.

SSPs are usually measured by a conductance, temperature and depth (CTD) system or a sound velocity profiler (SVP) system [10], and SSPs can also be obtained through inversion methods based on match field processing (MFP) [7], artificial neural networks (ANN) [5], and compressive sensing [3]. The original SSP is a sequence of discrete sound speed points, and if it is directly used for sound waves tracking, the ray theory calculation needs to be conducted between each two adjacent sound speed points, which requires too much time for calculation. Recently, the phenomenon of sound wave refraction is considered in some studies, and some localization correction methods based on ray tracing theory under varying sound speeds are put forward [8, 13]. Zhang et al. [13] adopts a single gradient linear sound speed approximation model, but it is hard to fit the real SSP curve accurately because the real SSP usually appears as S-type in shallow water and C-type in deep water; Liu et al. [8] assumes a three-layer linear SSP for localization, but how to choose suitable control points (linear anchors) and how many control points should be chosen for improving the approximation accuracy have not been discussed. Therefore, a simplified expression method of the SSP is necessary for reducing the calculation overhead while ensuring a certain accuracy of ray tracing. Essentially, the simplified expression of the SSP can be transformed into a curve contour extraction problem.

Polygon approximation criteria are proposed in $[9,12]$, such as the minimum mean square error (MSE), minimum closed area and maximum distance reduction. Ramer et al. [9] proposes an iterative sub-optimal depth-first binary tree searching (DFBTS) algorithm based on the maximum distance reduction criterion, where the candidate control points are traversed and selected in the sub-optimal interval during each iteration. The DFBTS has a low calculation cost, but an unreasonable distance threshold setting could result in non-uniform distribution of control points and larger SSP approximation errors. In [12], a global optimization searching method is proposed based on particle swarm optimization (PSO) according to the minimum MSE criterion. Although it shows better contour extraction performance in complex graphics, it brings high computational problems.

In this paper, we establish a stratified linear SSP simplification model to reduce the computational overhead of ray-tracing-based localization correction while guaranteeing good correction accuracy. We put forward a distance-minimization-based (maximum distance reduction) equal-interval control points searching (DMEICPS) algorithm for choosing control points of the stratified linear
SSP, which can accurately depict the real sound speed distribution over a large depth range with low searching time overhead.

The rest of this paper is organized as follows. The stratified linear SSP simplification model and the DM-EICPS algorithm is proposed in Sec. 2. Simulation results are discussed in Sec. 3, and conclusions are presented in Sec. 4 .

\section{STRATIFIED SSP SIMPLIFICATION METHOD}

\subsection{Stratified SSP Model}

The calculation of ray tracing is based on linear gradient SSPs. For original SSPs, one sound speed layer is constituted by two adjacent sampling points, so the computational time complexity of ray tracing is proportional to the number of SSP sampling points. As there are usually hundreds of sampling points for an original SSP, the ray tracing with the original SSP will cause huge computing time overhead. However, if some control points are selected and the original SSP is replaced by a stratified linear SSP based on these control points, the linear gradient layers of the original SSP will be greatly reduced, thus the computational time of ray tracing could be saved.

We establish a stratified SSP model to simplify the original SSP. Fig. 2 shows the stratified SSP model. By reasonably choosing the number and location of control points, the original SSP could be accurately approximated by the new stratified SSP, thus ensuring the accuracy of ray tracing.

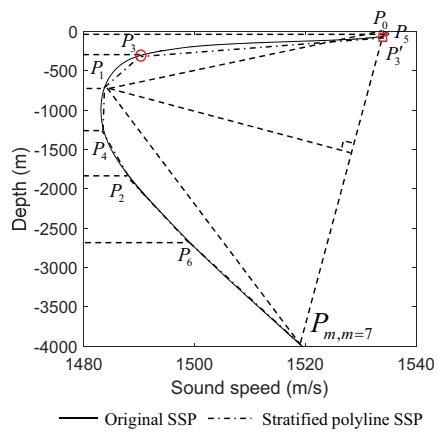

Figure 2: Stratified SSP model.

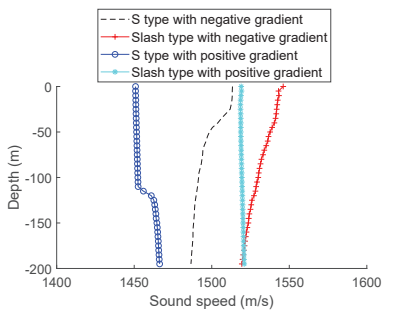

(a) Shallow-water SSPs

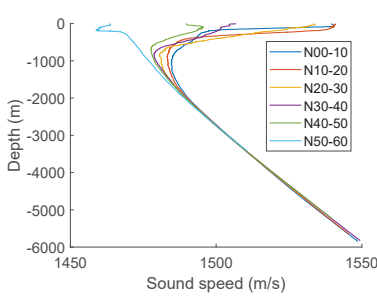

(b) Deep-water SSPs
Figure 3: Typical SSPs from 0-60 degrees north latitude of the Pacific Ocean. 


\subsection{DM-EICPS Algorithm}

To improve the approximating accuracy of the stratified linear SSP to the original SSP, we propose a DM-EICPS algorithm to find appropriate control points. Some typical types of SSPs sampled from 0-60 degrees north latitude of the Pacific Ocean are shown in Fig. 3 , which exhibit obvious shape characteristics. The shallow-water sound speed is mainly affected by temperature, and the SSP is $\mathrm{S}$-shaped or slash-shaped distribution with positive or negative gradient. The deep-water SSP changes with depth, showing a C-shaped or slash-shaped distribution. Regardless of the shallow-water or the deep-water SSPs, the curve can be approximated as a two-segment single-convex curve, thus the control point searching can be realized by one-dimensional optimization method. Since the SSP curve is not strictly convex, single-point searching method, such as binary searching, may fall into local optimal solutions, so we adopt an equal-interval searching method to find the optimal interval where the target control point locates. The detailed description of the proposed DM-EICPS is presented in Algorithm 1.

The DM-EICPS algorithm is designed as a global optimal solution searching algorithm. In each iteration, the candidate control point set is composed of several optimal points on sub-curves according to the maximum distance reduction criterion, then the global optimal solution is obtained by quadratic screening, so the final control points would not be unbalanced as [9]. However, the SSP curve is not strictly convex, thus the final result would be sub-optimal that close to the optimal result.

\subsection{Stratified SSP Based Ray Tracing Theory}

For the stratified linear SSP given by Algorithm 1, the total propagation time and horizontal propagation distance of the signal can be derived as a function of the glancing angle, which are presented in [5] as:

$$
\begin{gathered}
t=\sum_{u=1}^{M} \Delta t_{u}=\sum_{u=1}^{M}\left|\frac{1}{g_{o}^{u}} \ln \frac{\tan \left(\frac{\pi}{4}+\frac{\theta_{u-1}}{2}\right)}{\tan \left(\frac{\pi}{4}+\frac{\theta_{u}}{2}\right)}\right|, \\
x=\sum_{u=1}^{M} \Delta x_{u}=\frac{c\left(z_{0}\right)}{\cos \theta\left(z_{0}\right)} \sum_{u=1}^{M}\left|\frac{\sin \theta_{u-1}-\sin \theta_{u}}{g_{o}^{u}}\right|,
\end{gathered}
$$

where $\Delta t_{u}$ is the propagation time of the signal at the $u$ th depth layer, $g_{o}^{u}$ is the gradient of the linear SSP at the $u$ th depth layer, $\theta_{u}$ is the glancing angle of the signal at the depth of the $u$ th control point, $c\left(z_{0}\right)$ is the initial sound speed, $\theta\left(z_{0}\right)$ is the initial glancing angle, and $\Delta x_{u}$ is the horizontal propagation distance of the signal at the $u$ th depth layer. According to the Snell's law of refraction:

$$
\begin{aligned}
& c\left(z_{0}\right) \cos \theta_{u}=c_{u} \cos \theta\left(z_{0}\right), u=1,2, \ldots, M, \\
& n_{u} \cos \theta_{u}=n\left(z_{0}\right) \cos \theta\left(z_{0}\right), u=1,2, \ldots, M,
\end{aligned}
$$

where $c_{u}$ and $n_{u}$ are the sound speed and refractive index at the depth of the $u$ th control point, respectively; and the trigonometric relationship:

$$
\begin{gathered}
\sin \theta_{u}=\sqrt{1-\cos ^{2} \theta_{u}}, \\
\tan \left(\frac{\theta_{u}}{2}+\frac{\pi}{4}\right)=\frac{1+\sin \theta_{u}}{\cos \theta_{u}},
\end{gathered}
$$

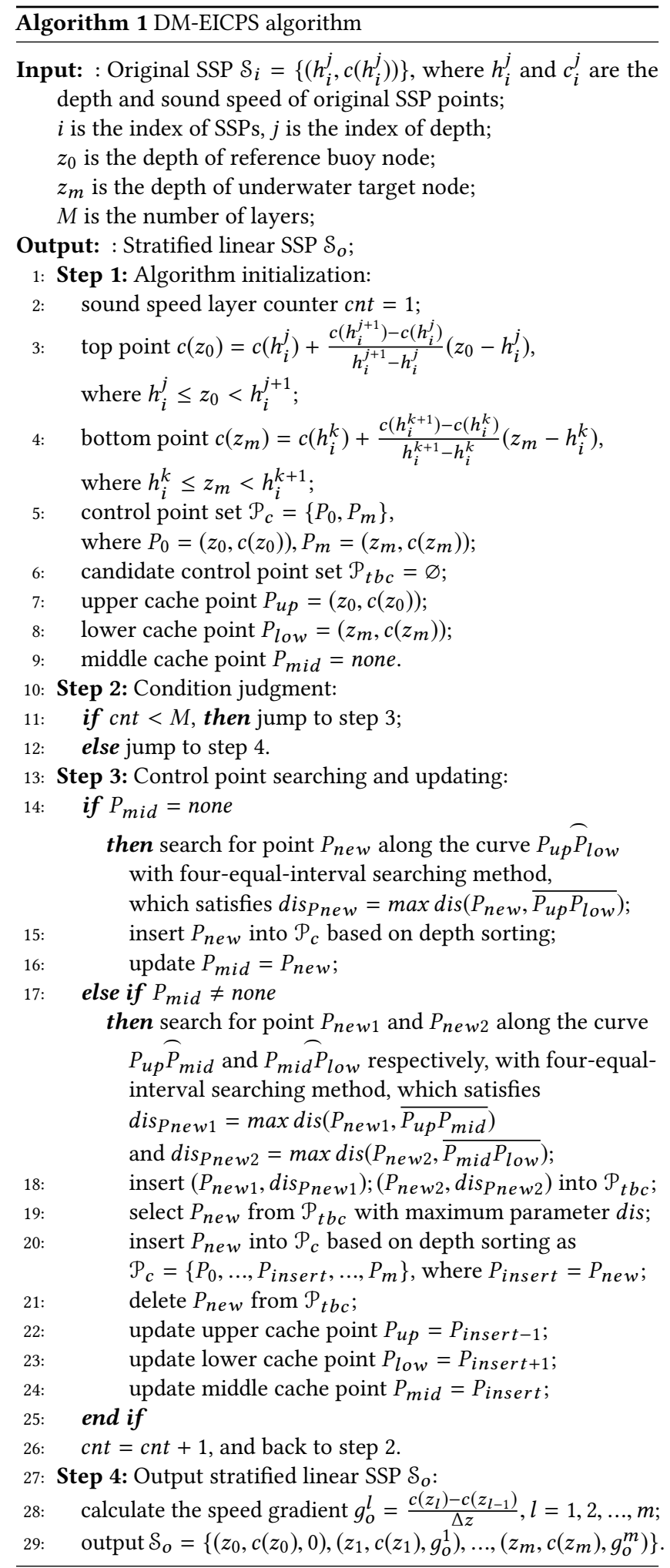

the signal propagation time (2) and horizontal propagation distance (3) can be modified as:

$$
x=\frac{c\left(z_{0}\right)}{\cos \theta\left(z_{0}\right)} \sum_{u=1}^{M}\left|\frac{\Delta z_{u}}{c_{u}-c_{u-1}}\left(\sqrt{\Gamma_{u-1}}-\sqrt{\Gamma_{u}}\right)\right|,
$$




$$
t=\sum_{u=1}^{M}\left|\frac{\Delta z_{u}}{c_{u}-c_{u-1}} \ln \left(\frac{c_{u}\left(1+\sqrt{\Gamma_{u-1}}\right)}{c_{u-1}\left(1+\sqrt{\Gamma_{u}}\right)}\right)\right|,
$$

where $\Gamma_{u}=1-\left(\frac{\cos \theta\left(z_{0}\right)}{c\left(z_{0}\right)}\right)^{2} c_{u}^{2}$. The formula (6) and (7) indicate that the total signal propagation time and horizontal propagation distance can be derived as functions of the initial glancing angle. Through formula (7), the signal initial glancing angle can be estimated when the calculated signal propagation time matches with the measured one, then the estimation value of horizontal propagation distance can be calculated by substituting the signal initial glancing angle into formula (6), so as to correct the ranging and localization results.

\subsection{Algorithm Complexity Analysis}

The time overhead of DM-EICPS algorithm mainly comes from the control point searching, the distance comparing of candidate points, and the new control point inserting (into the stratified linear SSP). Assume there are $N$ sampling points for an original SSP, and the number of stratified layers is $M(M-1$ control points). For the $m$ th $(m=1,2, \ldots, M-1)$ iteration, if $m=1$, the time complexity of the control point searching by the four-equal-interval searching method (three points searching) is $O\left(\log _{3} N\right)$, and the distance comparing is unnecessary because there is only one candidate point when $m=1$, which can be directly inserted into the stratified linear SSP between the first and end points; if $m>1$, the time complexity of the control point searching by the four-equal-interval searching method in the worst case is $O\left(2 \log _{3} \frac{(N-(m-1))}{2}\right)$, the time complexity of the candidate point distance comparing is $O\left(m \log _{2} m\right)$ by the quick sort algorithm, and the candidate point inserting time complexity is $O(m)$. Since the number of control points is much smaller than the points of original SSP, the total time complexity can be approximated as $O\left(\log _{3} N+2 \log _{3} \frac{(N-1)}{2}+\ldots+2 \log _{3} \frac{(N-(M-1))}{2}\right) \approx$ $O\left((2 M-1) \log _{3} N\right)$.

\section{SIMULATION RESULTS}

To verify the effectiveness of the proposed DM-EICPS algorithm, we make some simulation experiments on the SSP approximation accuracy, algorithm time complexity and localization correction effect. The depth information is obtained by pressure sensors, which is considered as prior information; and the node clock is assumed to be synchronized.

For the SSP approximation accuracy, we simplify 130 original SSPs (60 shallow-water SSPs sampled from 120-130 degrees west longitude, 30-40 degrees north latitude, and 70 deep-water SSPs sampled from 00-40 degrees north latitude at the Pacific Ocean) by the DM-EICPS algorithm with different numbers of layers, and results are compared with [9] $(0.2 \mathrm{~m} / \mathrm{s}$ distance threshold) and [12] (50 particles and iteration times).

Fig. 4 gives the comparison between stratified linear SSPs and original SSPs with 6 layers. The stratified linear SSPs generated by our DM-EICPS algorithm and the PSO searching algorithm can accurately express the original SSP; while control points of the SSP generated by the DFBTS algorithm [9] are non-uniformly distributed due to the inappropriate distance threshold setting, which reduces the SSP approximation accuracy.

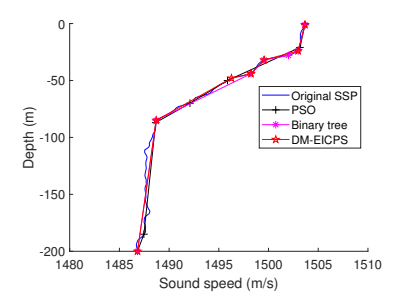

(a) Shallow-water SSPs

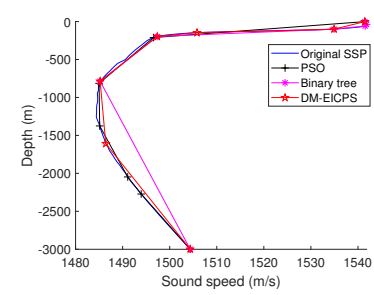

(b) Deep-water SSPs
Figure 4: Example of SSPs approximation.

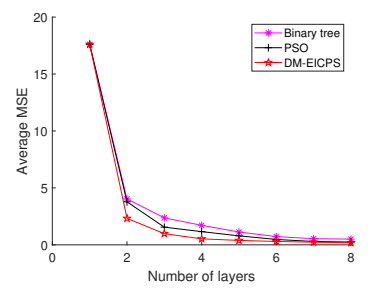

(a) Shallow-water SSPs

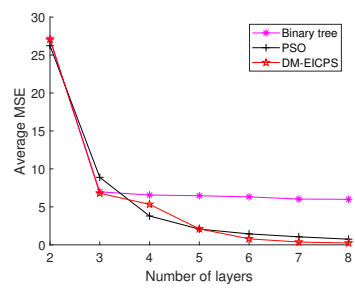

(b) Deep-water SSPs

\section{Figure 5: Average SSP approximation error comparison.}

Fig. 5 shows the average MSE of 130 SSPs with different numbers of layers. When the number of layers is equal to 1, the approximate SSPs obtained by different algorithms are all the same, which is a single gradient SSP between the transmitting node depth and the receiving node depth. As the number of layers increases, the DM-EICPS algorithm performs best in terms of the error reduction rate and the approximation accuracy.

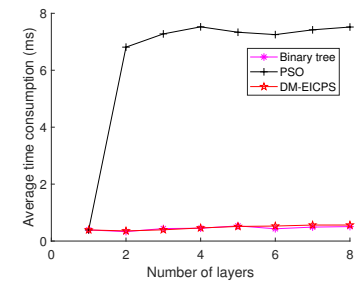

(a) Shallow-water SSPs

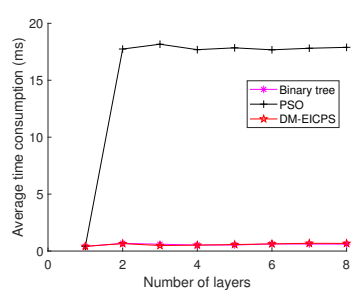

(b) Deep-water SSPs
Figure 6: Average time overhead comparison of SSP approximation.

To evaluate the time overhead performance of the DM-EICPS algorithm, the execution time on the above 130 SSPs with given layer numbers $1-8$ are tested.

Fig. 6 shows the average computational time of different SSP approximation algorithms. When the layer number is 1 , the SSP is obtained according to the sound speed of the depth where the transmitting node and the receiving node are located, thus the execution time of each algorithm is the same. However, as the layer number increases, the control point searching time of the DFBTS and our 


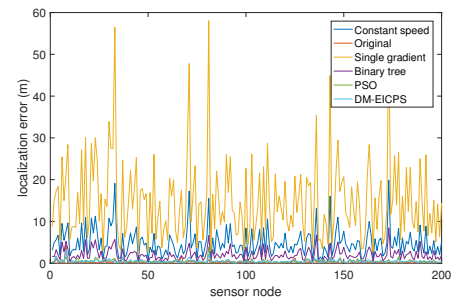

(a) Localization errors of 200 sensor nodes

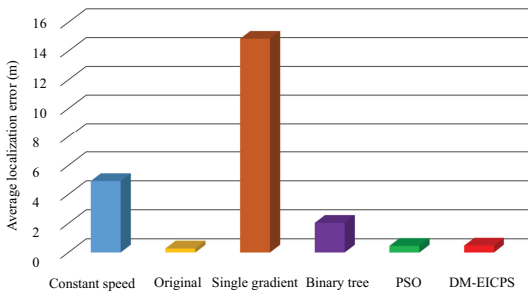

(b) Average localization error

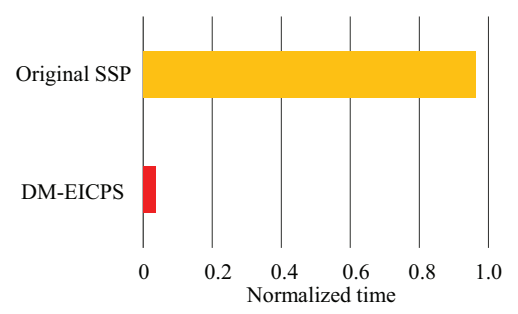

(c) Average localization time overhead

Figure 7: Comparison of localization accuracy and time overhead with and without ray-tracing-based localization corrections.

DM-EICPS algorithm is of the same order of magnitude, which is much smaller than the time consumption of the PSO searching algorithm.

To evaluate the SSP approximation effect on localization accuracy, we introduce the Monte Carlo method to locate randomly distributed underwater sensor nodes with a given SSP. The simulation area for localization test is set to be $10 \mathrm{~km} * 10 \mathrm{~km} * 3 \mathrm{~km}$, and the communication range of each node is $3.2 \mathrm{~km}$. We consider 200 nodes that are randomly distributed at the depth between $1000 \mathrm{~m}$ and $3000 \mathrm{~m}$ and 100 uniformly deployed buoy nodes over the water surface. The TOA is used for localization, and the time measurement error is Gaussian distributed with zero mean and standard deviation of $0.1 \mathrm{~ms}$.

Fig. 7 is the comparison of localization accuracy and time overhead with and without ray-tracing-based localization corrections, where Fig. 7 (a) presents detailed localization errors of 200 underwater sensor nodes in meters, Fig. 7 (b) gives the average localization error, and Fig. 7 (c) shows the normalized calculation time of ray tracing with the original SSP or the stratified linear SSP generated by DM-EICPS. The constant speed term is calculated by a linear propagation localization model at a sound speed of $1500 \mathrm{~m} / \mathrm{s}$. After ray tracing correction by the approximation SSP generated with the DFBTS, the PSO and the DM-EICPS algorithms, the localization error is reduced, and the location correction effect is better achieved with the SSPs of the PSO and the DM-EICPS algorithms. Through the comparison with Fig. 7 (b) and Fig. 7 (c), the localization correction with DM-EICPS-generated SSPs not only maintains good accuracy, but also significantly reduces the computational overhead.

\section{CONCLUSIONS}

Ray tracing is widely used in underwater positioning correction. To reduce the computational time complexity of ray tracing while maintaining good ray tracing accuracy, we establish a stratified linear SSP simplification model with a proposed low complexity DM-EICPS algorithm. The simulation results show that stratified SSPs obtained by DM-EICPS algorithm exhibits good approximation accuracy and low time overhead performance. With the simplified SSP, high localization accuracy and low computational complexity of ray tracing can be guaranteed at the same time.

\section{REFERENCES}

[1] P. Carroll, H. Mahmood, S. L. Zhou, H. Zhou, X. K. Xu, and J. H. Cui. 2014. OnDemand Asynchronous Localization for Underwater Sensor Networks. IEEE Transactions on Signal Processing 62, 13 (July 2014), 3337-3348. https://doi.org/ 10.1109/TSP.2014.2326996

[2] X. Z. Cheng, H. N. Shu, Q. L. Liang, and H. C. Du. 2008. Silent Positioning in Underwater Acoustic Sensor Networks. IEEE Transactions on Vehicular Technology 57, 3 (May 2008), 1756-1766. https://doi.org/10.1109/TVT.2007.912142

[3] Y. Choo and W. Seong. 2018. Compressive Sound Speed Profile Inversion Using Beamforming Results. Remote Sensing 10, 5 (April 2018), 704-722. https://doi. org/10.3390/rs10050704

[4] M. Erol, F. M. Vieira Luiz, and M. Geria. 2007. Localization with Dive 'N' Rise (DNR) beacons for underwater acoustic sensor networks. In WUWNet'07: Proceedings of the Second Workshop on Underwater Networks. ACM, Montreal, Quebec, Canada, 97-100. https://doi.org/10.1145/1287812.1287833

[5] W. Huang, D. S. Li, and P. Jiang. 2018. Underwater Sound Speed Inversion by Joint Artificial Neural Network and Ray Theory. In WUWNet'18: Proceedings of the Thirteenth ACM International Conference on Underwater Networks \& Systems. ACM, Shenzhen, Guangdong, China, 1-8. https://doi.org/10.1145/3291940.3291972

[6] F. B. Jensen, W. A. Kuperman, M.B. Porter, and H. Schmidt. 2011. Computational Ocean Acoustics (2nd. ed.). Springer, New York, NY. 3-4 pages.

[7] Z. L. Li, H. Li, R. H. Zhang, F. H. Li, Y. X. Yu, and L. Peng. 2015. Sound Speed Profile Inversion Using A Horizontal Line Array in Shallow Water. Science China Physics, Mechanics \& Astronomy 58, 1 (Jan 2015), 1-7. https://doi.org/10.1007/s11433014-5526-x

[8] J. Liu, Z. H. Wang, J. H. Cui, S. L. Zhou, and B. Yang. 2016. A Joint Time Synchronization and Localization Design for Mobile Underwater Sensor Networks. IEEE Transactions on Mobile Computing 15, 3 (March 2016), 530-543. https://doi.org/10.1109/TMC.2015.2410777

[9] U. Ramer. 1972. An Iterative Procedure for the Polygonal Approximation of Plane Curves. Computer graphics and image processing 1, 3 (1972), 244-256. https://doi.org/10.1016/S0146-664X(72)80017-0

[10] R. J. Urick. 1983. Principles of Underwater Sound (3rd. ed.). Tata McGraw-Hill Education, New York, NY. 88-90 pages.

[11] J. Yan, X. N. Zhang, X. Y. Luo, Y. Y. Wang, C. L. Chen, and X. P. Guan. 2018. Asynchronous Localization With Mobility Prediction for Underwater Acoustic Sensor Networks. IEEE Transactions on Vehicular Technology 67, 3 (March 2018), 2543-2556. https://doi.org/10.1109/TVT.2017.2764265

[12] P. Y. Yin. 2004. A Discrete Particle Swarm Algorithm for Optimal Polygonal Approximation of Digital Curves. fournal of Visual Communication and Image Representation 15, 2 (2004), 241-260. https://doi.org/10.1016/j.jvcir.2003.12.001

[13] B. B. Zhang, H. Y. Wang, L. M. Zheng, J. F. Wu, and Z. W. Zhuang. 2017. Joint Synchronization and Localization for Underwater Sensor Networks Considering Stratification Effect. IEEE Access 5, 3 (October 2017), 26933-26943. https://doi. org/10.1109/ACCESS.2017.2778425

[14] Z. Zhou, Z. Peng, J. H. Cui, Z. J. Shi, and A. C. Bagtzoglou. 2011. Scalable Localization with Mobility Prediction for Underwater Sensor Networks. IEEE Transactions on Mobile Computing 10, 3 (March 2011), 335-348. https://doi.org/ 10.1109/TMC.2010.158 


\section{A HEADINGS IN APPENDICES}

A.1 Introduction

A.2 Stratified SSP Simplification Model

A.3 Simulation Results

A.4 Conclusions

A.5 References 